\title{
MAGNETOTRANSMISSION MEASUREMENTS OF INTRA-SHALLOW-DONOR TRANSITIONS IN SEMI-INSULATING GaAs*
}

\author{
K. Karpierz, K. Kożuchowski and M. Grynberg \\ Institute of Experimental Physics, Warsaw University \\ Hoża 69, 00-681 Warszawa, Poland
}

In this paper we present the results of an investigation of the $1 s-2 p_{+}$ intra-shallow-donor transition by means of an extremely difficult magnetotransmission experiment performed on semi-insulating GaAs. We report the temperature dependence of the transition intensity. We noticed the absence in the absorbance spectra of a well-pronounced structure which is observed at low magnetic fields in photoconductivity measurements. The results are discussed in terms of a fluctuating potential from ionized centres in semi-insulating GaAs.

PACS numbers: 72.40.+w, 78.20.Ls, 78.50.Ge

Shallow donors in semi-insulating (SI) GaAs are subjected to strong local electric fields due to compensation and weak screening [1]. At liquid helium temperatures, these centres are not occupied by electrons due to the pinning of the Fermi level on the deep native donor EL2 [2,3]. Applying a previously reported method [2] - external infrared illumination (with wavelength $\lambda=0.86 \mu \mathrm{m}$ ) we are able to create a measurable, steady population of neutral shallow donor states. The samples ${ }^{\dagger}$ are then additionally illuminated with far infrared (FIR) light in order to obtain intra-shallow-donor transitions. The transmission of FIR light $(\lambda=119 \mu \mathrm{m})$ was measured by a homemade, very sensitive bolometer as a function of applied magnetic field. The experimental set-up allows us to measure the transmission through the sample and its photoconductivity simultaneously. All experiments were made at low temperatures - near $4.2 \mathrm{~K}$.

*This work is supported in part by the Project No 202659101 of the Committee for Scientific Research .

TThe sample was a rectangular slab $(3.0 \times 1.0 \times 0.5 \mathrm{~mm}$ ) of bulk SI GaAs (grown by the LEC technique in the (100) direction) with a concentration of the EL2 defect about $10^{16} \mathrm{~cm}^{-3}$. The dark resistance at $4.2 \mathrm{~K}$ was of the order of $10^{12} \Omega$. Two non-rectifying (down to $4.2 \mathrm{~K}$ ) $\mathrm{Au}-\mathrm{Ge}-\mathrm{Ni}$ contacts were alloyed by Dr. Kaminiska and Dr. Piotrowska from the Institute of Electron Technology in Warsaw. 
Previous photoconductivity experiments $[2,4]$ showed a significant difference in the behaviour of intra-shallow-donor transitions in the SI material with respect to $n$-type GaAs (see Fig. 1b). In this paper a typical absorbance spectrum (derived from transmission) for a SI sample is presented on Fig. 1a (upper curve). It is practically the same as in $n$-type GaAs (lower curve). The well-pronounced peak corresponds to the $1 s-2 p_{+}$transition in the hydrogen-like [5] shallow-donor system, split by an external magnetic field. For magnetic fields outside of the peak

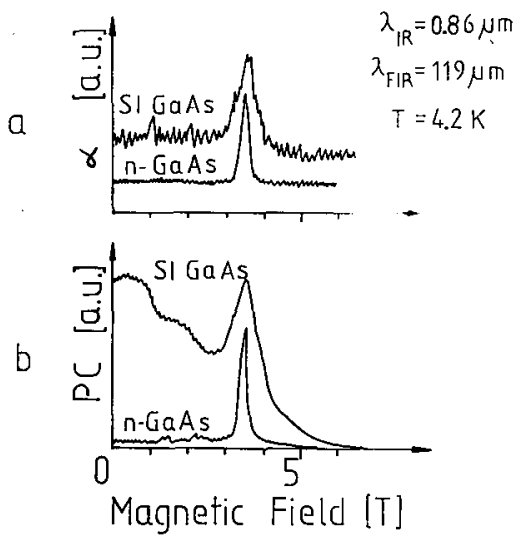

Fig. 1. a) Absorbance of FIR radiation in $n$-type and SI GaAs samples versus magnetic field. Both curves have different vertical scales. b) Photoconductivity of $n$-type and SI GaAs samples due to FIR illumination versus magnetic field. Vertical scales for both curves are not the same.

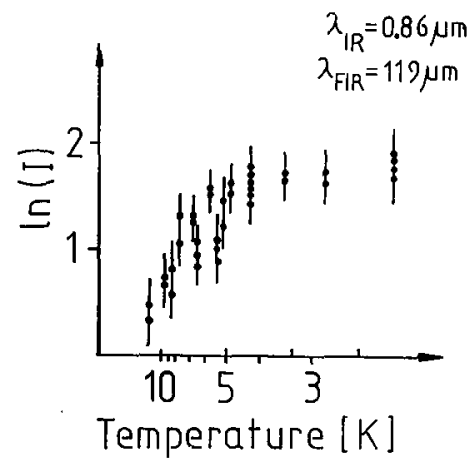

Fig. 2. Temperature dependence of the $1 s-2 p_{+}$intra-shallow-donor transiton intensity for SI GaAs.

region no comparable absorbance was visible. Figure 2 shows the results of temperature investigations in the $1 s-2 p_{+}$transiton intensity. With increasing temperature we observe a decrease in the transition intensity due to the temperature depopulation of shallow-donor ground state. From the saturation value for the lowest 
temperatures we are able to determine the concentration of neutral (i.e. populated by electrons) shallow-donor centres. It is of the order of $10^{13} \mathrm{~cm}^{-3}$. Comparing this value with a typical concentration of shallow-donor centres (a few times $10^{15}$ $\mathrm{cm}^{-3}$ ) we find that about every thousandth shallow centre is occupied by an electron. Matching this ratio with information that samples were illuminated by low intensity infrared light coming from a monochromator we sec a relatively huge metastability. We attribute this result to the influence of a fluctuating potential on the lifetime of electrons in the shallow-donor ground state.

Introducing a probability $P_{\alpha}$ of transferring an electron from the ground state of a shallow-donor to the excited state and a probability $P$ of transferring an elcctron from the excited state of the shallow-donor to the continuum of Landau levels, we can describe the photoconductivity $(\mathrm{PC})$ and transmission $(\mathrm{T})$ signals as follows [2]:

$$
\mathrm{PC} \propto P_{\alpha} P,
$$

$\mathrm{T} \propto P_{\alpha}$.

Comparing the upper and lower curves in Fig. 1a we see that for both materials the absorbance spectra (derived from transmission) are similar - the fluctuating potential does not affect $P_{\alpha}$ very strongly. However, the PC spectra are completely different from each other. For lower magnctic fields there is an enhanced PC signal in SI GaAs (comparable with the $1 s-2 p_{+}$transition) which docs not appear in the $n$-type. The stronger photoconductive signal must come from a larger value of the probability $P$. The only difference between the two samples is the presence of local electric fields in the SI material. Therefore, we conclude that the fluctuating potential strongly influences the probability $P$.

\section{References}

[1] J. Łusakowski, R. Merten, M. Grynberg, Acta Phys. Pol. A 82, (1992).

[2] K. Karpierz, M.L. Sadowski, M. Grynberg, Acta Phys. Pol. A 80, 291 (1991).

[3] M. Kaminiska, Rev. Phys. Appl. 23, 793 (1988).

[4] K. Karpierz, M.L. Sadowski, Acta Phys. Pol. A79, 121 (1991).

[5] J. Simola, J. Virtamo, J. Phys. B 11, 3309 (1978). 